\title{
THE TREATMENT OF LOBAR PNEUMONIA WITH PENICILLIN ${ }^{1}$
}

\author{
By WILLIAM S. TILLETT, JAMES E. MCCORMACK, AND \\ MARGARET J. CAMBIER \\ (From the Department of Medicine of New York University College of Medicine and the \\ Third Medical Division of Bellevue Hospital, New York City)
}

(Received for publication January 18, 1945)

This communication, which deals with the use of penicillin in the treatment of lobar pneumonia, and an accompanying one (1) describing the local treatment of pneumococcal empyema with penicillin, are extensions and elaborations of an earlier report (2) on the same subjects. The material embodied in the earlier article consisted of 46 cases of pneumonia and 8 cases of pneumococcal empyema. In the present report, 64 additional cases of pneumonia are included, and, in the accompanying article, 13 additional cases of empyema have been added, making total numbers of 110 pneumonias and 21 empyemas, respectively, that have been assembled for presentation.

The results obtained with the use of penicillin in pneumonia were derived from cases that were available for treatment during two successive years, consisting of the seasons of $1942-43$ and 1943-44. Data for the two periods are given separately in Table $I$ and are also consolidated as total results.

Since the study has been primarily concerned with cases of pneumococcal etiology, other cases of acute pulmonary infections in which the bacteriology was indeterminate and which, in addition, presented various clinical and roentgenological findings of ill-defined suppurative and nonsuppurative preumonitis, have been excluded from the present analysis.

\section{Serological types of pneumococci}

The incidence of infections caused by the serological types of pneumococci, Types I to VIII, which most frequently give rise to severe pneumonia, are comparable for the two periods recorded in Table I. In the $1942-43$ series, 30 out

1 The penicillin was provided by the Office of Scientific Research and Development from supplies assigned by the Committee on Medical Research for clinical investigations recommended by the Committee on Chemotherapeutic and Other Agents of the National Research Council. of 46 cases were due to the particular pneumococcal types mentioned and in the 1943-44 series 41 out of 64 cases were caused by the same types, the number in each instance representing approximately two-thirds of the total. The most definite difference in the type incidence during the two separate years is to be noted in the occurrence of 17 cases of Type II pneumococcus pneumonia during $1943-44$ as contrasted with 6 similar cases in the preceding year.

The number of cases listed bacteriologically as "unclassified" refers to instances in which the sputum contained pneumococci but the organisms were not identified either by quellung reaction or by mouse inoculation as belonging to Types I to XXXII. The patients listed as having "no sputum" were selected for inclusion in the pneumococcal group of pneumonias on the basis of a characteristic history and classical clinical findings, including leukocytosis. It seems not unlikely that if the typing of sputum from these cases had been accomplished they would have distributed themselves proportionately among the usual types.

\section{Bacteremia}

The fact that the initial blood culture was positive in 40 ( 36.3 per cent) instances indicates that the cases selected for treatment were particularly suitable for assaying the effect of the therapy. The incidence of bacteremia in the 1943-44 period was somewhat higher (39 per cent) than in the preceding year (32.6 per cent). The difference is accounted for by the 17 cases of Type II pneumococcal infection encountered during 1943-44 of which 10 (58 per cent) yielded positive blood cultures.

Response to penicillin therapy. A definite response to treatment with penicillin has been arbitrarily defined as occurring when fever and accompanying symptomatology either rapidly disappeared during the first 12 to 24 hours or signifi- 
TABLE I

Results obtained from the treatment of pmeumonia with penicillin

\begin{tabular}{|c|c|c|c|c|c|c|c|c|c|c|c|c|c|c|c|}
\hline \multirow{3}{*}{$\begin{array}{c}\text { Pneumo. } \\
\text { Types }\end{array}$} & \multicolumn{5}{|c|}{ Cases of $1942-43$ series } & \multicolumn{5}{|c|}{ Cases of 1943-44 series } & \multicolumn{5}{|c|}{ Combined results } \\
\hline & \multirow{2}{*}{$\begin{array}{l}\text { No. } \\
\text { cases }\end{array}$} & \multirow{2}{*}{$\begin{array}{c}\text { Posi- } \\
\text { tive } \\
\text { blood } \\
\text { cul- } \\
\text { ture }\end{array}$} & \multicolumn{3}{|c|}{ Response to penicillin } & \multirow{2}{*}{$\begin{array}{l}\text { No. } \\
\text { cases }\end{array}$} & \multirow{2}{*}{$\begin{array}{l}\text { Posi- } \\
\text { tive } \\
\text { blood } \\
\text { cul- } \\
\text { ture }\end{array}$} & \multicolumn{3}{|c|}{ Response to penicillin } & \multirow{2}{*}{$\begin{array}{l}\text { No. } \\
\text { cases }\end{array}$} & \multirow{2}{*}{$\begin{array}{c}\text { Posi- } \\
\text { tive } \\
\text { blood } \\
\text { cul- } \\
\text { ture }\end{array}$} & \multicolumn{3}{|c|}{ Response to penicillin } \\
\hline & & & $\begin{array}{c}\text { Defi- } \\
\text { nite }\end{array}$ & $\begin{array}{c}\text { Indefi- } \\
\text { nite }\end{array}$ & Died & & & $\begin{array}{c}\text { Defi- } \\
\text { nite }\end{array}$ & $\begin{array}{c}\text { Indefi- } \\
\text { nite }\end{array}$ & Died & & & $\begin{array}{c}\text { Defi- } \\
\text { nite }\end{array}$ & $\begin{array}{c}\text { Indefi- } \\
\text { nite }\end{array}$ & Died \\
\hline $\begin{array}{c}\text { II } \\
\text { III } \\
\text { IV } \\
\text { V } \\
\text { VI } \\
\text { VII } \\
\text { VIII } \\
\text { IX } \\
\text { XI } \\
\text { XII } \\
\text { XV } \\
\text { XIX to XXIX } \\
\text { Unclassified } \\
\text { No sputum }\end{array}$ & $\begin{array}{r}11 \\
6 \\
1 \\
1 \\
5 \\
3 \\
4 \\
1 \\
2 \\
1 \\
1 \\
3 \\
4 \\
3\end{array}$ & $\begin{array}{l}4 \\
3 \\
1 \\
1 \\
1 \\
2 \\
2 \\
2 \\
0 \\
\\
0 \\
0 \\
0 \\
1 \\
0 \\
0\end{array}$ & $\begin{array}{r}10 \\
6 \\
1 \\
1 \\
4 \\
3 \\
3 \\
3 \\
\\
2 \\
1 \\
1 \\
2 \\
3 \\
2\end{array}$ & $\begin{array}{l}1 \\
1 \\
1\end{array}$ & $\begin{array}{l}1 \\
1\end{array}$ & $\begin{array}{r}6 \\
17 \\
5 \\
2 \\
2 \\
1 \\
5 \\
3 \\
1 \\
\\
3 \\
1 \\
2 \\
7 \\
9\end{array}$ & $\begin{array}{r}3 \\
10 \\
1 \\
1 \\
2 \\
0 \\
2 \\
2 \\
1 \\
\\
1 \\
1 \\
0 \\
1 \\
0\end{array}$ & $\begin{array}{r}6 \\
16 \\
4 \\
2 \\
2 \\
3 \\
3 \\
1 \\
1 \\
2 \\
1 \\
1 \\
6 \\
7\end{array}$ & $\begin{array}{l}1 \\
2\end{array}$ & 1 & $\begin{array}{r}17 \\
23 \\
6 \\
3 \\
7 \\
1 \\
8 \\
7 \\
1 \\
1 \\
2 \\
4 \\
2 \\
5 \\
11 \\
12\end{array}$ & $\begin{array}{r}7 \\
13 \\
2 \\
2 \\
3 \\
0 \\
4 \\
4 \\
0 \\
1 \\
0 \\
1 \\
1 \\
1 \\
1 \\
0\end{array}$ & $\begin{array}{r}16 \\
22 \\
5 \\
3 \\
6 \\
6 \\
6 \\
6 \\
1 \\
2 \\
3 \\
2 \\
3 \\
9 \\
9\end{array}$ & $\begin{array}{l}2 \\
1 \\
3\end{array}$ & $\begin{array}{l}1 \\
1 \\
1 \\
1\end{array}$ \\
\hline Total & 46 & 15 & 39 & 4 & 3 & 64 & 25 & 54 & 6 & 4 & 110 & 40 & 93 & 10 & 7 \\
\hline
\end{tabular}

cantly and progressively defervesced during the first 2 days. In other cases for which penicillin was also of definite value but in which the effect was not reflected by rapid changes in the course of the temperature, significant improvement was evident by the clearing of bacteremia and by considerable alleviation of distressing symptomatology such as general intoxication, cyanosis, and labored respirations. The latter group usually comprised the most severe infections and in spite of the fact that fever did not rapidly abate, their recovery indicated particularly the value of penicillin in pneumonia.

Ninety-three (84.6 per cent) of the 110 patients were considered to have exhibited a definite response to treatment with penicillin. In 71 (76 per cent) of the 93, the rate of recovery was both complete and abrupt as reflected in the fact that their temperatures reached normal limits within the first day or two following the institution of treatment. Thirteen of the patients who responded in this manner had bacteremia on admission.

As stated in the previous article (2), the rapidity with which the temperature fell to normal ranges following penicillin therapy was often a striking phenomenon, the suppression of the infection not infrequently becoming evident within the first 12 to 15 hours.
In the remaining 22 ( 24 per cent) of the 93 patients who responded favorably to penicillin, fever persisted beyond the period of treatment. Twenty of the 22 in this group had bacteremia and many were admitted late in the disease. They either had complications referable to the pneumococcal infection itself or their pneumonia was superimposed on some other pre-existing disorder. In spite of these circumstances, their recovery from pneumonia was complete.

The acute complicating factors which delayed recovery in the latter group of 22 severe cases were as follows: empyema-2 cases, sterile pleural effusion-3 cases, purulent arthritis-2 cases, delayed resolution-3 cases, co-existing pulmonary diseases such as bronchiectasis, asthma, or possibly primary atypical pneumonia- 6 cases, purpura - 1 case, leukemia - 1 case, gangrene of the foot- 1 case, salpingitis- 1 case, and unexplained fever of 10 days' duration-2 cases.

On the basis of our present experience, if a patient believed to have pneumococcal pneumonia fails to respond to penicillin therapy with a significant drop in fever by the third day of treatment, it has been found important to review again several aspects of the case such as the bacterial etiology, the possible development of some pneumococcal complication, or the possible presence 
of an additional intercurrent disorder that may account for retardation of recovery.

The capacity of penicillin to eradicate bacteremia has been found to be one of the most striking effects. Of the forty cases (36.3 per cent) of the whole series which had bacteremia on admission, in every instance irrespective of the ultimate outcome, the second blood culture, taken usually on the following day, was sterile.

A limited number of observations have been made on the rapidity with which the circulating organisms were eradicated. In 5 cases, repeated blood cultures were taken at 3-hourly intervals after each intravenous injection of penicillin. The rapidity with which the cultures became sterile was as follows: in 2 instances, 3 hours after the first intravenous dose of 25,000 units of penicillin; in 2 instances, 3 hours after the second dose; and in 1 instance, 3 hours after the third dose. The progressive decrease in the number of organisms in the blood stream appears to indicate the bactericidal action of penicillin, in vivo, comparable to the findings observed in vitro (3).

In Table $I, 10$ of the recovered cases have been listed as exhibiting an indefinite response to penicillin therapy. None of the group had bacteremia and none of them were seriously ill. They pursued febrile courses for 7 to 14 days with only gradual improvement in spite of the administration of presumably adequate amounts of penicillin.

Complications such as pleurisy, bronchiectasis, or pulmonary fibrosis, which might account for the equivocal response, were suspected of being present but were not definitely established. The explanation of the failure of this group to respond remains, therefore, obscure.

Deaths. Seven patients who received penicillin therapy died (mortality rate 6.3 per cent). One of the patients of the 1943-44 series included in the fatal group was moribund on admission and died 7 hours later. A second patient, also of the 1943-44 series, was admitted to our wards with pneumococcal (Type VIII) meningitis which had developed in association with an earlier pneumonia that had been treated with sulfadiazine.

If the 2 cases of the $1943-44$ series just mentioned are excluded, 5 deaths remain as a basis for estimating a mortality rate of 4.5 per cent.

Details of the 3 fatal cases of the 1942-43 series were given in the previous article (2). Of the remaining 2 deaths among the $1943-44$ series, the circumstances were as follows: one of the patients was a 65-year-old woman with an indeterminate history. She had pneumonia, bacteremia, and purulent arthritis due to pneumococcus, Type XII. Auricular fibrillation was present. She had received 145,000 units of penicillin before exitus. A blood culture taken on the second day was sterile. She died 48 hours after admission without responding to treatment.

The second recent fatal case was a 42-year-old white male who was admitted on the 4th day of pneumonia due to pneumococcus, Type II. Bacteremia was present. $\mathrm{He}$ received a total of 400,000 units within the first 9 hours, 100,000 units being given intravenously every 3 hours for 4 doses. The dosage which he received represented part of a study directed toward attempting to determine whether or not relatively massive doses of penicillin given over a short period of time would be as efficacious as small doses extended over several days. $\mathrm{He}$ received a total amount of penicillin greater than that employed according to usual procedures. On the day following admission, he appeared improved and a second blood culture was sterile. Consequently, subsequent treatment was temporarily withheld. On the following day, however, he became worse. Penicillin therapy was reinstituted but the patient died 6 hours later.

This patient, as will be subsequently pointed out in a discussion of dosage and duration of treatment, illustrates the importance of a continuation of treatment over several days in relation to day of disease on which treatment was first instituted rather than the total quantity of penicillin administered during a brief period in severely ill patients.

\section{Cases with pneumococcal complications}

Although additional studies of pneumococcal empyema will be the subject of a separate communication (1), 2 cases are included in the present series since they were first seen during the acute pneumonic phase of the disease. They were treated by injections of penicillin both intravenously and intramuscularly. Bacteremia, which was present in each instance on admission, cleared within the first 24 hours. They ultimately recovered. 
Whether or not empyema developed in spite of the otherwise successful administration of penicillin is not clear. The acute pneumonic signs during the first few days of observation overshadowed the possible evidence of the presence of empyema. In view of the fact that the patients were admitted on the 5th and 8th days, respectively, of severe pneumonia, it seems possible that the suppurative infection of the pleura was present before therapy was begun. Up to the present time, in our experience, neither the intravenous nor intramuscular administration of penicillin has been found to be of value in the treatment of empyema after it has been established.

In the present series of cases, the incidence of 2 cases of empyema (1.8 per cent) among 110 cases of pneumonia is lower than the rate of 5 per cent which is usually given as representing the frequency of empyema as a complication of pneumonia before the modern forms of specific chemotherapy were adopted.

Four cases of purulent arthritis complicating acute bacteremic pneumonia were encountered in the present series. The arthritis was present on admission and was bilateral in each of the cases. Knees, ankles, or wrists, or combinations of these sets of joints, were affected.

Pneumococci, Types I (2 cases), VIII, and XII, were isolated from the sputum, blood, and the purulent articular exudates.

One of the cases, as mentioned previously, terminated fatally.

The remaining 3 recovered from the acute pneumonia. With respect to the arthritis, one of them recovered completely after several weeks of gradual defervescence; in another, in whom the acute articular infection was superimposed on an extensive pre-existing osteoarthritis, the joints returned to their earlier status; in the third patient, destruction of articular tissues occurred with permanent disablement which has required prolonged orthopedic treatment.

It is interesting to note that in each of the 3 cases which recovered, the arthritic exudate became sterile following the intravenous injection of penicillin. With specimens of exudate from 2 of them, tests in vitro were performed which demonstrated the presence of active penicillin that had diffused from the blood stream. Other investigators (4) have also recently reported that penicillin administered intravenously was found to diffuse into inflamed joints.

The 3 patients in whom delayed resolution occurred were admitted to the hospital on the 7th, 8 th, and 10th days of disease, respectively. Their illness was severe and was accompanied by bacteremia. Among the patients in general; however, delayed resolution has not been found to occur as a characteristic of pneumonia treated with penicillin.

Dosage of penicillin. In the previous report (2), a scheme of dosage was developed which was based on observations derived from experimentally planned trials with respect to quantity of penicillin per dose, frequency of dosage, and duration of treatment. The treatment which was outlined may be summarized as follows:

For cases of moderate severity, the injections for each day consisted of 10,000 units per dose given intramuscularly for 4 doses and the continuation of the procedure was conditioned by the day of disease on which treatment was begun. In patients admitted within 3 days of the onset, treatment was continued for at least 4 days; in patients admitted after the 3rd day of illness, 3 consecutive days of treatment often proved sufficient.

For cases severely ill, the first 2 doses consisted of 25,000 units, given intravenously at 3hour intervals, followed, also at the same interval, by 2 doses of 10,000 units, given intramuscularly for the first day. When a favorable response was evident, the subsequent daily treatment consisted of 4 doses of 10,000 units each, given intramuscularly at 3-hour intervals for each day that treatment was continued.

The procedure as outlined obviates the necessity of giving injections of penicillin constantly throughout each 24-hour period since the remissions evoked by a series of 4 injections were found to endure for 20 to 24 hours after the last dose. However, in view of the fact that patients were often admitted during the evening or night the injections of the second day followed the treatments of the first day as a continuous period of 8 injections.

According to this regime the total amount of penicillin per case varied from 120,000 to 190,000 units and treatment lasted for 3 to 4 days.

In reviewing the dosage of the cases of the 
1943-44 series, an analysis reveals that of 15 cases with bacteremia the average amount per case was 248,000 units and of 15 cases without bacteremia, the average amount per case was 175,000 units. The two factors which accounted for the recent increases in dosage were a greater number of intravenous injections (4 to 8 in sequence) to severe cases and a tendency to extend treatment beyond the third or fourth day when appreciable degrees of fever persisted. Analyzed objectively, no very clear cut advantage was demonstrable from the increased dosage except such advantage as might be referable to insuring sufficient therapy for the patients who were seriously ill.

In the previous communication (2), details of individual cases were given in which treatment with moderate amounts of penicillin (10,000 units every 3 hours for 4 doses) were injected for a single day only. Among the cases so treated in which the illness was relatively mild, the brief period of therapy proved sufficient. However, in other patients in whom therapy was suspended after the administration of the first 40,000 units, the primary remission, which lasted approximately 24 hours, was followed by a relapse requiring further treatment.

Additional observations have been made with regard to the effectiveness of a single day of treatment by administering large doses on the day of admission. One patient, treated in this manner, received on the first day a total of 300,000 units given intravenously in 3 individual doses of 100,000 units each at 3 -hour intervals. The prompt fall in temperature and symptomatic improvement which followed endured for 30 hours. A sharp exacerbation of the infection then developed which was controlled when penicillin therapy was resumed.

A second case, similarly treated for a single day, was described among the fatal cases. As stated earlier, he received 400,000 units on the day of admission.

Although each of these patients received larger total amounts of penicillin than the average quantity given even to severe cases, the failure of the single day method emphasizes the greater importance of continuation of therapy rather than the size of individual doses. The rôle of immunity developed by the patient appears to be an impor- tant factor in transforming the remission of infection induced by the drug into permanent cure.

On the basis of our additional experience, therefore, it seems likely that the earlier recommendations remain essentially valid as a basis for assaying the amount of penicillin necessary to suppress the infection. However, the method of handling unusually serious cases encountered late in the disease may invite the use of large initial doses $(25,000$ to 100,000 units intravenously for the first day or two) for the purpose of eradicating the bacteremia as rapidly as possible and thus preventing metastatic complications such as meningitis, pericarditis, or endocarditis from developing.

Infections caused by pneumococci resistant to sulfadiazine treated with penicillin. Among the patients included in the present study, the infections in 3 instances were proved by laboratory tests to be caused by pneumococci that possessed a high degree of resistance to the antibacterial action of sulfadiazine. The serological types of the infecting strains were Types I, VII, and VIII, respectively. Mice, infected with the strains, died following injections of 10 to 100 M.L.D. in spite of treatment with amounts of sulfadiazine that were, in tests with other strains of pneumococci of a similar type, sufficient to effect cure.

In each of the patients from whom the resistant strains were derived, appreciable blood levels of sulfadiazine resulting from the immediately previous treatment were present concomitantly with a bacteremia. Prompt improvement leading to recovery followed penicillin therapy in each case.

Since the patients did not respond to the initial treatment with sulfadiazine, their courses suggested that the infecting pneumococci may have been sulfonamide-fast from the beginning of the disease rather than having acquired the property during treatment.

Similar conditions to those just described were presented in an earlier article (5) in which 2 patients having, bacteremic pneumonia due to sulfonamide-fast pneumococci were successfully treated with type specific antipneumococcus serum.

Cases in which penicillin therapy was replaced by the administration of sulfadiazine. In 8 of the 10 patients (listed in Table I) who exhibited no appreciable response to penicillin, sulfadiazine was subsequently administered during the febrile 
period. In 7 of them the change in treatment was without effect. None of the patients were seriously ill. They all recovered gradually by lysis.

In one patient from whose sputum pneumococcus Type III was isolated, the change in treatment from penicillin, which seemed ineffectual, to sulfadiazine was followed by prompt recovery. Bacteremia was not present in the case.

Since the sulfadiazine was first administered on the 9th day of disease, the possibility that spontaneous crisis may have occurred coincident with the change in therapy requires consideration in interpreting the results.

The patient's strain was not tested for fastness to penicillin. In this connection, it may be stated that among 20 different strains of pneumococci derived from patients, none have been encountered which exhibited by laboratory tests an appreciable degree of resistance to penicillin. Up to the present time, a review of the literature fails to reveal the occurrence of penicillin-fast strains of pneumococci derived from patients.

\section{Summary}

This report contains the results obtained from the treatment of 110 cases of pneumococcal pneumonia with penicillin during the seasons of 1942-43 and 1943-44.

The total mortality ( 7 cases) was 6.3 per cent or, if corrected as indicated in the text of the article, 4.5 per cent.

Among the patients who recovered, the effectiveness of penicillin was deemed to be definite in 84.6 per cent (93 cases) and to be equivocal in 9.1 per cent (10 cases).

Bacteremia, which occurred in 40 of the patients (36.3 per cent), was successfully eliminated in each case and this result constitutes one of the most striking effects of penicillin therapy in pneumonia.

With respect to dosage of penicillin, emphasis has been placed on extension of treatment over several days ( 3 to 5 ) depending on the day of disease on which therapy is begun.

Repetition of injections continuously throughout each 24 hours of treatment has not, except in seriously ill individuals, appeared necessary or advantageous.

The total amounts of penicillin employed have ranged from 120,000 to 200,000 units in cases of moderate severity, and from 150,000 to 400,000 units in serious cases, depending on the evidence of response noted in the first 24 to 48 hours of treatment.

In 3 instances, infections caused by sulfonamidefast pneumococci were effectively treated with penicillin.

No toxic reactions referable to penicillin have been noted.

\section{BIBLIOGRAPHY}

1. Tillett, W. S., McCormack, J. E., and Cambier, M. J., The use of penicillin in the local treatment of pneumococcal empyema. J. Clin. Invest., 1945, 24, 595.

2. Tillett, W. S., Cambier, M. J., and McCormack, J. E., The treatment of lobar pneumonia and pneumococcal empyema with penicillin. Bull. New York Acad. Med., 1944, 20, 142.

3. Hobby, G. L., Meyer, K., and Chaffee, E., Observations on the mechanism of action of penicillin. Proc. Soc. Exper. Biol. and Med., 1942, 50, 281.

4. Herrell, W. E., Nichols, D. R., and Heilman, D. R., Penicillin: its usefulness, limitations, diffusion, and detection. J. A. M. A., 1944, 125, 1003.

5. Tillett, W. S., Cambier, M. J., and Harris, W. H., Sulfonamide-fast pneumococci. J. Clin. Invest., 1943, 22, 249. 